\title{
Failure rates associated with guided versus non-guided dental implant placement: a systematic review and meta-analysis
}

\author{
Nancy Abdelhay ${ }^{1,2}$, Soni Prasad ${ }^{3}$ and Monica Prasad Gibson (iD ${ }^{4 凶}$ \\ (c) The Author(s) 2021
}

OBJECTIVE: The purpose of the systematic review and meta-analysis was to evaluate implant failure rates and their association with guided and free-hand implant placement techniques.

MATERIALS AND METHODS: A literature search was conducted across PubMed, Medline via Ovid, Cochrane database, and Google Scholar. The search was completed in September 2020. Series of meta-analyses were conducted to compare implant failure rates with guided and free-hand techniques.

RESULTS: A total of 3387 articles were identified from the electronic search. After applying the inclusion criteria, eight articles were selected for qualitative assessment and four for quantitative synthesis (meta-analysis). The included studies had a risk ratio of 0.29 $(95 \% \mathrm{Cl}: 0.15,0.58), P<0.001$ for the use of guided implant placement. Implant failure rates were affected by the different placement techniques indicated by the test for overall effect $(Z=3.53, P=0.0004)$. The incidence of implant failure in guided surgery versus free-hand surgery was found to be $2.25 \%$ and $6.42 \%$, respectively.

CONCLUSION: Both guided and free-hand implant placement techniques resulted in a high implant survival rate. However, implant failure rates were almost three times higher in the free-hand implant placement category. A guided implant placement approach is recommended for a successful outcome.

BDJ Open (2021)7:31 ; https://doi.org/10.1038/s41405-021-00086-1

\section{INTRODUCTION}

Prosthodontic rehabilitation with dental implants requires accurate implant placement for predictable functional, and aesthetic outcomes. ${ }^{1,2}$ Implant dentistry has developed numerous advancements in technology, materials, techniques, and concepts to achieve the desired beneficial clinical results. ${ }^{3}$

Implant placement is a prosthetically driven procedure and requires thorough restorative and surgical treatment planning. ${ }^{4}$ The patient's anatomy, medical condition, practitioner experience, and surgical approach are factors that ultimately influence the outcome. ${ }^{5,6}$ There are several surgical techniques utilized during implant placement. ${ }^{7}$ A surgeon can place an implant either freehand, with a pilot drill guide, or with a fully guided system. ${ }^{8,9} \mathrm{~A}$ fully guided template is fabricated with the help of a cone-beam computed tomography $(\mathrm{CBCT})$ and related implant planning software. 10,11 This process involves a "crown-down" approach that visualizes an ideal prosthetic position to orient the virtual implant placement. ${ }^{12,13}$ A surgical guide is then fabricated to replicate this planned position. ${ }^{14}$ The CBCT also provides an indepth understanding of the underlying bone morphology, anatomy, and location of vital structures. ${ }^{2,15}$

Recently, a dynamic navigation system has been introduced to aid in implant placement. This technology uses intraoperative optical tracking of the hand-piece position with installed cameras to guide the surgeon in "real-time," thus providing visual feedback on a screen. ${ }^{16}$ However, this review will focus on the comparison between free-hand and fully guided implant placements only.

The fully guided implant placement that utilizes the computergenerated guide is more accurate than traditional surgical guides and free-hand placements. ${ }^{17}$ Several excellent studies and reviews have outlined the accuracy of computer-generated guides with respect to anatomical landmarks and range of error. ${ }^{18-20}$ However, not many reviews have investigated implant failures.

Once an implant is placed, the outcome of its placement is dependent on distinct clinical and radiographic parameters that determine the implant's success or survival. ${ }^{21}$ A "successful" implant is defined as an implant remaining in situ and free of all biological and technical complications over the entire observation period. ${ }^{22}$ According to Smith and Zarb, implant success criteria include the absence of mobility, peri-implant radiolucency, pain, and infection. In addition, annual bone loss should be $<0.2 \mathrm{~mm}$ after the first year of service along with the satisfactory appearance of implant prostheses. They proposed a minimum success rate of $85 \%$ at the end of 5 years and $80 \%$ at the end of 10 years. ${ }^{23}$ On the other hand, implant survival refers to the implant remaining in situ at the follow-up examination. ${ }^{22,24}$

The goal of surgical implant placement is to have a high success rate. However, a surviving implant that does not meet all the success criteria is sometimes an acceptable (but not ideal) outcome. Early implant failure resulting primarily from inaccurate

\footnotetext{
${ }^{1}$ Faculty of Medicine and Dentistry, Department of Dentistry, University of Alberta, Edmonton, AB, Canada. ${ }^{2}$ Faculty of Dentistry, Alexandria University, Alexandria, Egypt. ${ }^{3}$ Graduate Prosthodontics, Marquette University School of Dentistry, Milwaukee, WI, USA. ${ }^{4}$ Faculty of Medicine and Dentistry, Division of Periodontology, University of Alberta, Edmonton, AB, Canada. ${ }^{\bowtie}$ email: mgibson@ualberta.ca
} 
planning or surgical complications causes angst among patients and surgeons alike. ${ }^{25}$ Also, previously failed implant sites are at a higher risk for future implant placement. ${ }^{26}$ Therefore, attempts should be made to avoid early implant failures. Implant success and survival rely on the osseointegration between the implant surface and surrounding bone. ${ }^{27}$ Lack or loss of osseointegration results in implant failures. ${ }^{28,29}$ Early implant failures occur before prosthetic loading and are seen in 1-2\% of patients within the first few weeks. ${ }^{30}$ Late failures usually occur after prosthetic loading and are seen in about $5-10 \%$ of patients. ${ }^{31}$ Peri-implantitis, factors affecting the microbial environment, and prosthetic rehabilitation are common causes for late implant failure. ${ }^{32}$

While several excellent reviews have compared the success and survival rates between free-hand versus fully guided implant placement, very few have evaluated the failures. ${ }^{28,29,32-34}$ Researchers have correlated possible early and late implant failure risk factors with age, sex, smoking, ${ }^{35}$ type of edentulism, bone quality and volume, ${ }^{36}$ implant location, diameter, length, ${ }^{37}$ immune factors, and various systemic diseases. ${ }^{38}$ Although computer-guided implant placement is predictable, its performance with respect to failure rates must be critically evaluated and compared with free-hand implant placement. This systematic review aimed to evaluate the association between implant failure rates and surgical placement using fully guided and free-hand techniques.

\section{MATERIALS AND METHODS \\ Study selection}

A literature search was performed in September 2020 across four electronic databases: PubMed and Medline via Ovid (1990 to September 2020), Cochrane database (Wiley, September 2020), and Google Scholar. No time or language restrictions were applied to attain the maximum number of results regarding implant dentistry. Manual record search across dental journals and other relevant databases generated literature more specific to the review focus and undiscovered from the above primary databases. Several combinations of keywords like dental implants, guided, non-guided, free-hand, three-dimension, peri-implantitis, and risk factors, were used during the search process to generate records pertaining to implant failure and use of surgical guides. Primary screening involved examining the title and abstract of generated records. Full-text studies that seemed to meet the criteria were included and further assessed. Two reviewers independently carried out the secondary screening of the remaining records involving full-text assessment of study methods, results, and discussions. The final selection of studies was made by a discussion between authors under PICO-based inclusion/exclusion criteria.

\section{Inclusion, exclusion criteria and study design (PICO)}

The Population of interest included all patients in need of implant placements and patients referred/scheduled for implant surgery. General inclusion criteria were healthy adults $>18$ years of age who were nonsmokers or light smokers $(<5$ cigarettes a day). Interventions were oral implant placements. The Comparison involved the usage of guided versus free-hand implant placements. Experimental studies directly pertaining to the Outcome in question were examined for data extraction. Included study types were randomized controlled trials (RCTs), clinical controlled trials (CCTs), and prospective/retrospective clinical studies. For the meta-analysis, included study designs were RCTs and CCTs. Outcomes of interest were (1) the incidence of early implant failure using either technique and (2) associated risk factors. Subsequently, patients were excluded if they were immunocompromised, $<18$ years of age, pregnant, or had systemic disease. Studies without treatment interventions such as reviews, case reports, and commentaries were excluded. Finally, studies were excluded if they did not report on implant failure outcomes or had different populations of interest.

\section{Assessment based on Quality Appraisal of Reliability Studies (QAREL) Checklist}

The risk of bias assessment for included RCTs and cohort studies was performed following the guidelines by Cochrane systematic review handbook..$^{39,40} \mathrm{JBI}$ Critical Appraisal Checklist for Quasi-Experimental
Studies for non-randomized and randomized experimental studies was used. $^{41}$

The QAREL Checklist 28 was used on the included studies to identify their reliability according to 11 items. The QAREL Checklist provided a quality assessment for the spectrum of participants and examiners, examiner blinding, order effects of examination, suitability of the time interval among repeated measurements, appropriate test application and interpretation, and appropriate statistical analysis. The checklist also helped assess the variability in performance and reporting of physical examination procedures. Each criterion was framed as a question and had response options of definitely yes (low risk of bias), probably yes, probably no, and definitely no (high risk of bias). The included studies' quality was classified based on QAREL scores: a score of $67 \%$ or more indicated high quality, $50-66 \%$ moderate quality, and $<50 \%$ low quality.

\section{Data extraction}

The data extraction process was executed in duplicate and independently by two authors. It was then double-checked between two authors to validate the gathered information. Any disagreements were resolved by discussion or consultation with the third author.

\section{Data synthesis and statistical analysis}

The meta-analysis was performed using RevMan 5.3, constructing a forest plot with $l^{2}$ statistics to analyze and present variability due to heterogeneity among the gathered studies. Relative weights of included studies were expressed in percentages, and the risk ratio (RR) with $95 \%$ confidence interval $(\mathrm{Cl})$ was computed per study or subgroup.

\section{RESULTS}

\section{Literature search}

A total of 3403 potentially relevant titles and abstracts were found by the electronic search and additional evaluation of reference lists. During the first screening, 2079 publications were excluded after duplicates were removed. Another 1890 records were excluded based on the title and keywords, and abstract evaluation. In addition, 189 full-text articles were thoroughly evaluated. A total of 181 papers were excluded because they did not fulfill the systematic review's inclusion criteria (Fig. 1). Eight articles were included in the qualitative assessment and four in quantitative synthesis (meta-analysis).

\section{Description of studies}

A detailed summary of the included studies is presented in Table 1. Data extraction identified three RCTs $s^{4,42,43}$ and five cohort studies. ${ }^{6,44-47}$ All studies stated the number of implants placed using guided versus free-hand techniques and the investigation outcomes. Five studies ${ }^{4,43,45-47}$ identified implant failure as it is related to guided or free-hand implant placement. One study investigated the malpositioning of implants placed using either mucosa- or bone-supported guides and free-hand placement. ${ }^{44}$ One RCT study investigated free-hand implant placement with preoperative $\mathrm{CBCT}$ and postoperative periapical radiograph. ${ }^{6}$ Another study compared free-hand and guided implant placement by prosthodontists and maxillofacial dentists. ${ }^{42}$ Five studies investigated implant failure rates using any of the techniques. Two studies did not specify the number of failed implants. ${ }^{15,24}$

\section{Assessment based on QAREL Checklist}

The included studies' methodological quality and reliability were assessed using the QAREL Checklist (Tables 2 and 3). The total ratings of the methodological quality of reliability or RCTs studies ranged between 50 and $100 \%$. Among the three studies included, two studies were of high quality (Q 100\%), and one study was of moderate quality (Q 50\%). The risk of bias within the article is presented in Table 2. The total ratings of the methodological quality of reliability for Cohort studies ranged between 36 and $90 \%$. Among the five studies included, three studies were of low quality (Q 36 and 54\%); one was of moderate quality (Q 63\%), and 


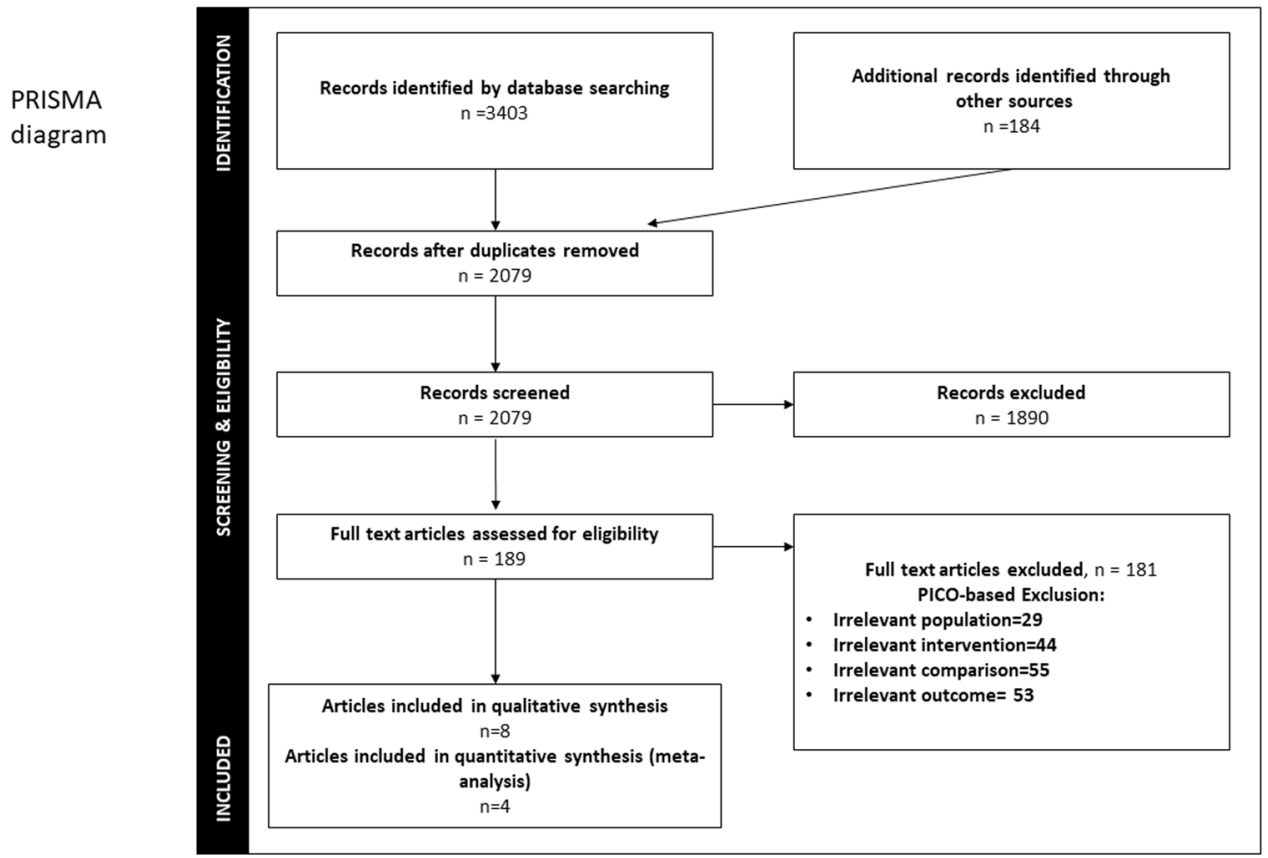

Fig. 1 PRISMA flow diagram of literature search strategy: including identification, screening, eligibility examination, and final inclusion. The number of records identified during the initial search represents the sum of all papers collected through each electronic database.

one was of high quality (Q 90\%). The risk of bias within the article is presented in Table 3 .

The forest plot analysis of included RCTs and cohorts is exhibited in Fig. 2. Three studies had insufficient data regarding the number of failures; hence the meta-analysis incorporated only five studies. The included studies had a RR of $0.29(95 \% \mathrm{Cl} 0.15$, $0.58), P<0.001$ for the use of guided implant placement. The forest plot exhibited medium heterogeneity $\left(I^{2}=38 \%\right)$. Implant failure rates were affected by the different placement techniques as indicated by the test for overall effect $(Z=3.53, P=0.0004)$. According to the results, the incidence of implant failure in guided surgery versus non-guided is $2.25 \%$ and $6.42 \%$, respectively. There was no statistically significant heterogeneity observed $\left(I^{2}=38 \%\right)$.

\section{DISCUSSION}

The specific aim of this systematic review was to compare failure rates of implants placed using a computer-generated guide and freehand placement. Guided placement resulted in accurate implant positioning in terms of parallelism between implants and less mesiodistal and buccolingual deviation. ${ }^{6,42,44,45}$ Although the accuracy in implant placement and survival rates using the two techniques have been reported extensively in the literature, there is limited information on implant failure rates with the two approaches.

Yogui et al. compared survival rates between computer-guided and freehand placement. They concluded that both techniques yielded a similar result. ${ }^{48}$ Also, Pozzi et al., in their review, suggested that survival rates of guided surgery were similar to conventional freehand protocols. ${ }^{4}$ According to a systematic review by Schneider et al., computer-guided implant placement had higher implant survival rates ranging from 91 to $100 \%$ after 12-60 months of follow-up. ${ }^{49}$

This systematic review found a significant difference in the failure rates between the two techniques. Eight studies met the inclusion criteria, and out of these, four were included in the metaanalysis. Meta-analysis indicated that the RR for guided implant placement was $0.29(0.15,0.58) p<0.001$. The incidence of implant failure in freehand surgery was almost three times higher than the guided protocol. Despite the overall low heterogeneity $\left(I^{2}=38 \%\right)$ among included studies, some variations existed in the follow-up duration, secondary interventions, and implant surgery types. This is not an uncommon finding in systematic reviews.

The fully guided technique presents an advantage of accuracy in implant placement when compared to the freehand technique. An accurate implant placement ensures a predictable restorative outcome. One disadvantage of a fully guided approach is that it involves additional cost, and in cases of limited mouth opening, following a fully guided drill sequence can be challenging. ${ }^{48}$ In addition, few studies have reported that guided surgeries can easily cause operator oversight during osteotomy preparation resulting in inadequate irrigation during surgery. ${ }^{48}$ This could interfere with bone healing and compromise the outcome. ${ }^{6,48}$ However, the finding of this systematic review was contrary to the suggested outcome.

In addition, some studies compared intraoperative and postoperative complications and morbidity following implant placement. ${ }^{46,47}$ In contrast to other studies, the present study reported reduced postoperative morbidity in terms of swelling, pain, and bleeding with guided implant placement compared to the freehand approach. ${ }^{4,43}$ Proper case selection and surgical execution could contribute to these differences.

One limitation that could be noted from the included studies was the differences in the operators' clinical experience and skill set. Another limitation was a low number of quality studies comparing guided to freehand implants. These observations call for the need for more standardized RCTs to establish the evidence, estimate the effect size, and standardize the potential effect modification in using guided and freehand implant placement techniques.

\section{CONCLUSION}

Within the limitations of this systematic review, both guided and freehand implant placement have a high implant survival rate. However, based on the results, implant failure rates were almost three times higher in freehand placement. 


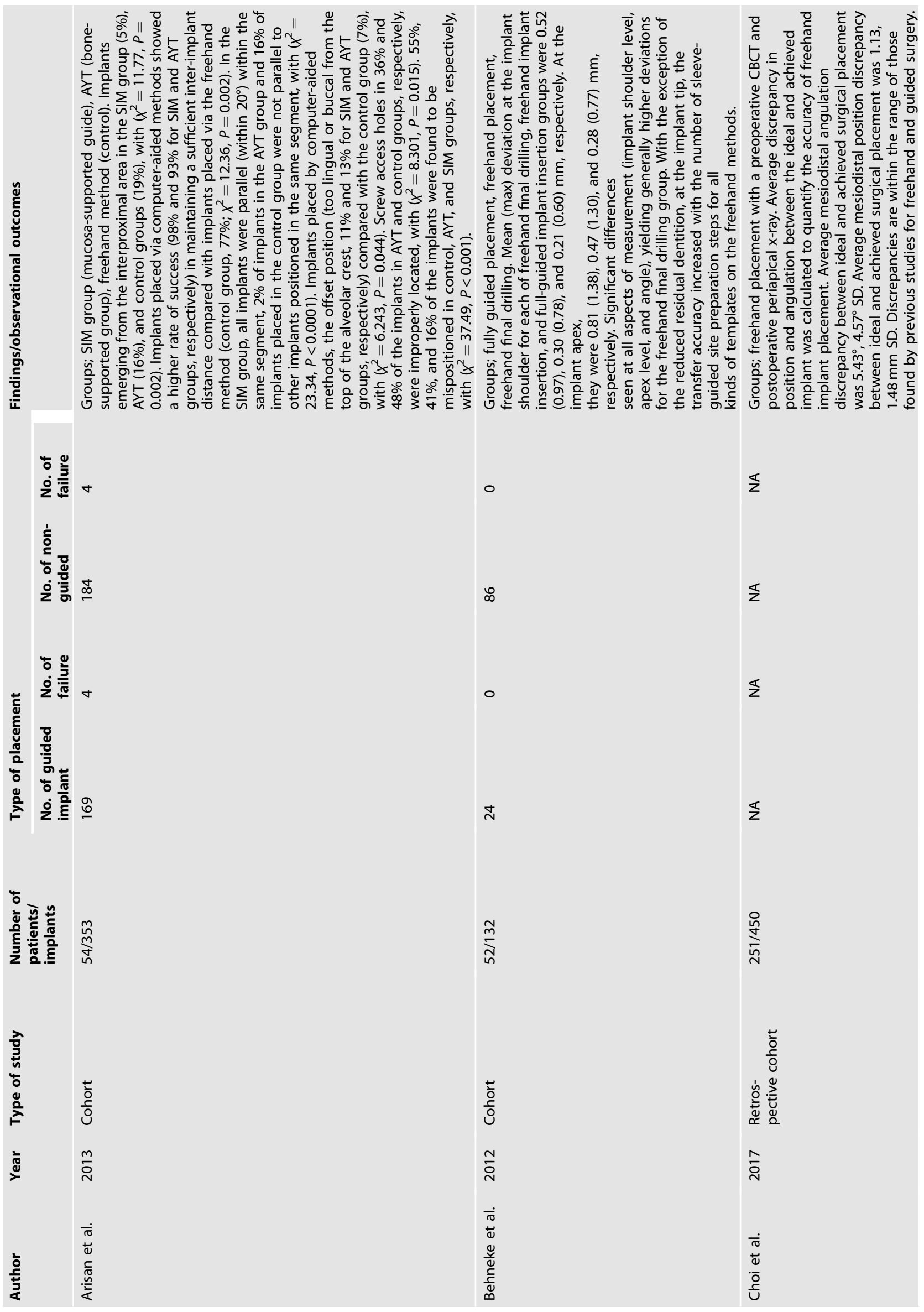



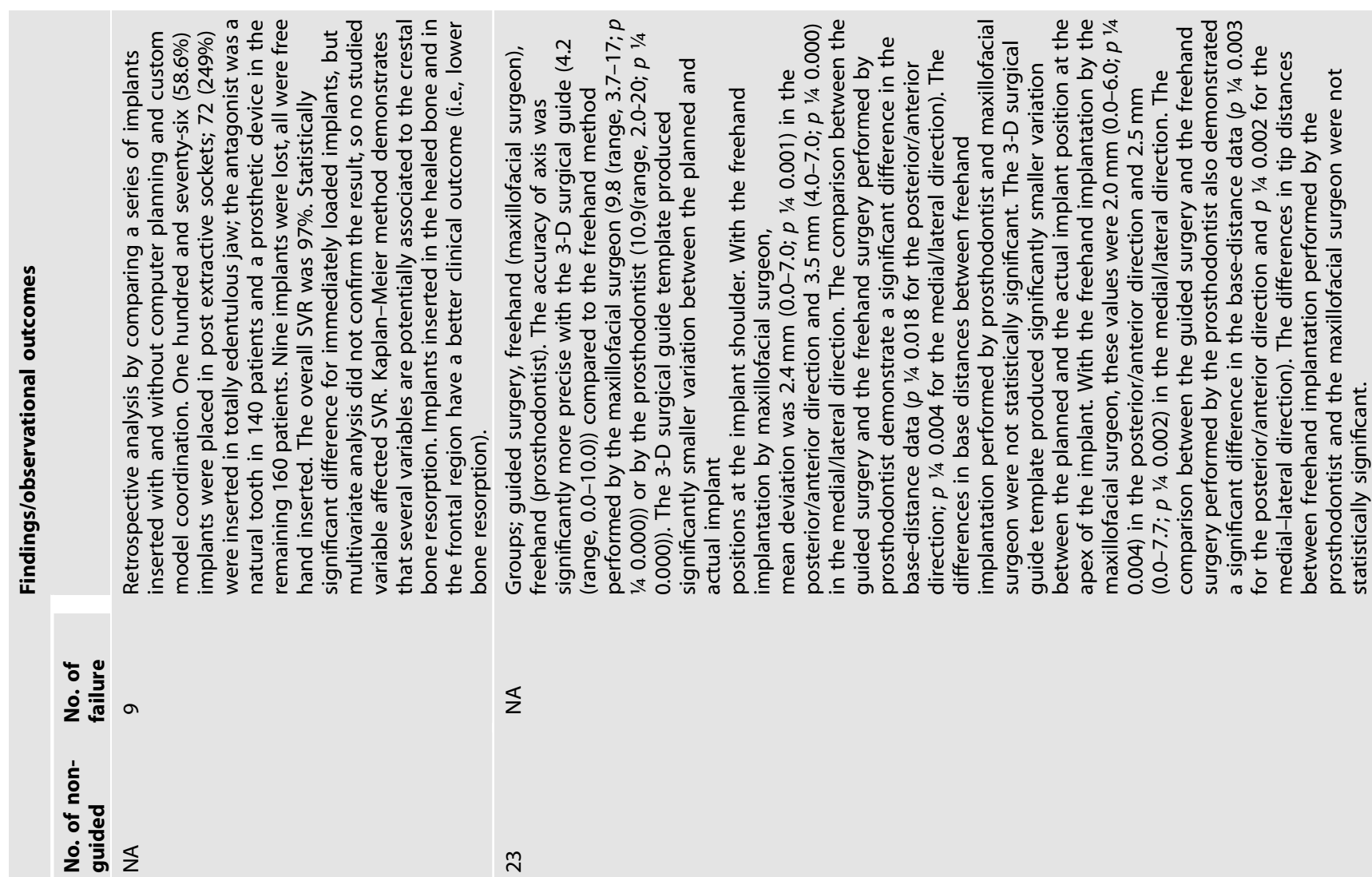

N

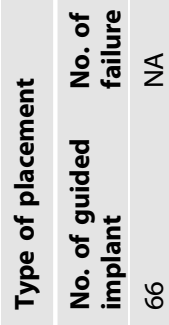

$\Sigma$

N

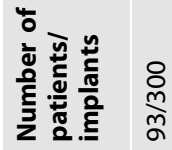

$\stackrel{\circ}{\circ}$

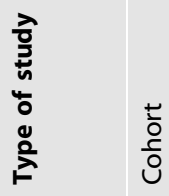

t্

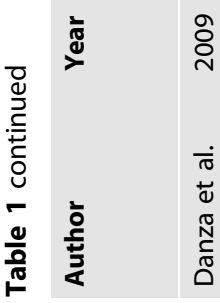

음

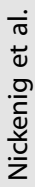




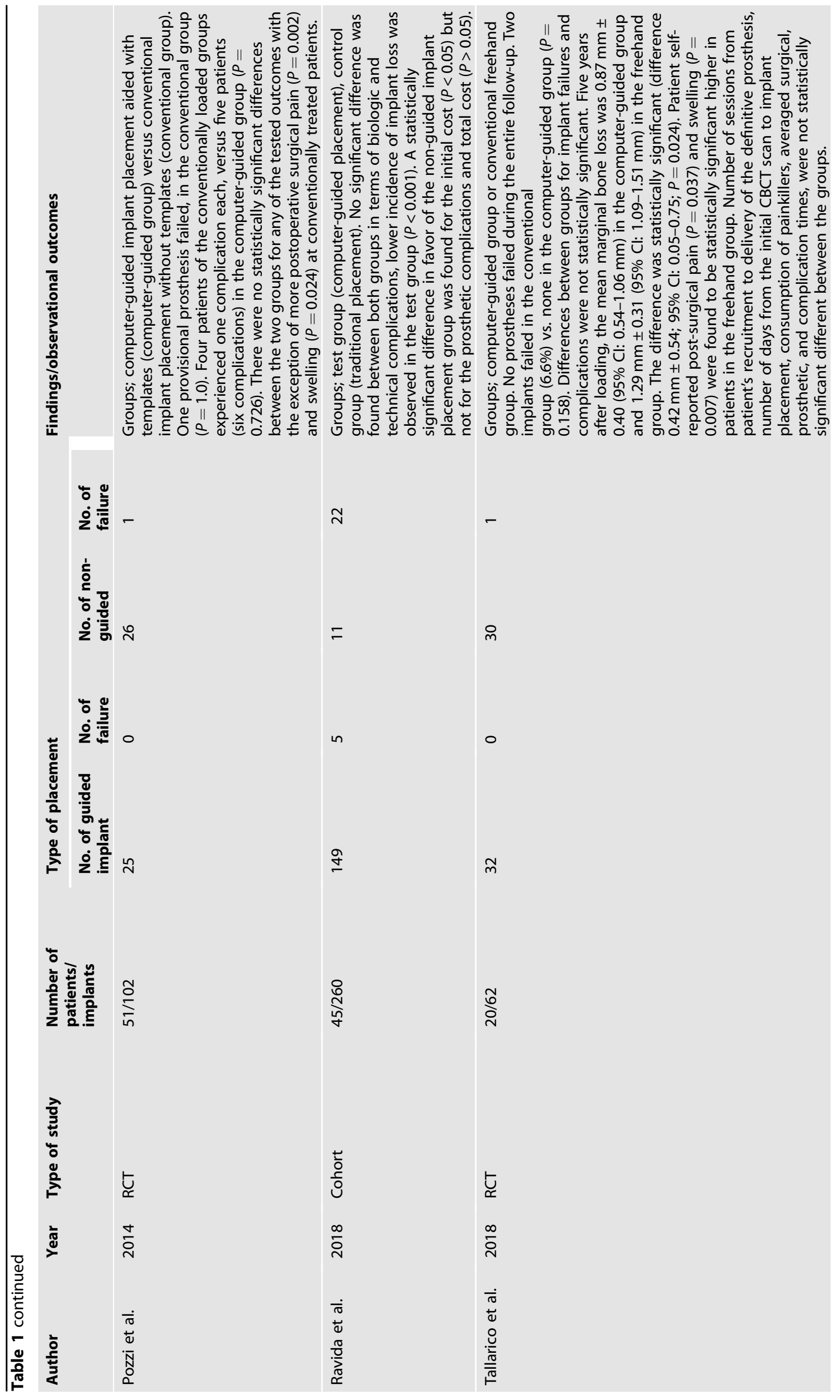


Table 2. Individual risk of bias assessment for controlled randomized studies: review authors' judgments about each risk of bias factor among the selected studies.

\section{Quality analysis of randomized clinical studies}

\begin{tabular}{|c|c|c|}
\hline Nickeing 2010 & Pozzi 2014 & Tallarico 2018 \\
\hline$y$ & $y$ & $y$ \\
\hline u & $y$ & $y$ \\
\hline$n$ & y & y \\
\hline y & y & y \\
\hline y & $y$ & $y$ \\
\hline $\mathrm{u}$ & $y$ & $y$ \\
\hline u & y & y \\
\hline y & y & $y$ \\
\hline y & y & $y$ \\
\hline $50 \%$ & $100 \%$ & $100 \%$ \\
\hline
\end{tabular}

Is it clear in the study what is the "cause" and what's the "effect" (i.e., there is no confusion about which variable comes first)?

Were the participants included in any comparisons similar?

Were the participants included in any comparisons receiving similar treatment/care, other than the exposure or intervention of interest?

Was there a control group?

Were there multiple measurements of the outcome both pre and post the intervention/ exposure?

Was follow up complete and if not, were differences between groups in terms of their follow up adequately described and analyzed?

Were the outcomes of participants included in any comparisons measured in the same way?

Were outcomes measured in a reliable way?

Was appropriate statistical analysis used?

Overall appraisal:

$50 \%$

$100 \%$

$100 \%$

Seven domains were analyzed for each record, and final judgments were made by discussion between authors.

$y$ yes, $n$ no, $u$ unclear.

Table 3. Individual risk of bias assessment for cohort studies: review authors' judgements about each risk of bias factor among the selected studies.

\section{Quality analysis of cohort studies}

Were the two groups similar and recruited from the same population?

Were the exposures measured similarly to assign people to both exposed and unexposed groups?

Was the exposure measured in a valid and reliable way?

Were confounding factors identified?

Were strategies to deal with confounding factors stated?

Were the groups/participants free of the outcome at the start of the study (or at the moment of exposure)?

Were the outcomes measured in a valid and reliable way?

Was the follow up time reported and sufficient to be long enough for outcomes to occur?

Was the follow up complete, and if not, were the reasons to loss to follow up described and explored?

Were strategies to address incomplete follow up utilized?

Was appropriate statistical analysis used?

Total \%

Seven domains were analyzed for each record, and final judgments were made by discussion between authors.

$y$ yes, $n$ no, $u$ unclear.

\begin{tabular}{|c|c|c|c|c|}
\hline Danza 2009 & Behneke 2012 & Arisan 2013 & Choi 2017 & Ravida 2018 \\
\hline $\mathrm{u}$ & $y$ & y & $\mathrm{u}$ & $y$ \\
\hline y & $y$ & $y$ & u & $y$ \\
\hline$y$ & $y$ & $y$ & $y$ & $y$ \\
\hline $\mathrm{n}$ & $\mathrm{n}$ & $\mathrm{n}$ & $y$ & $y$ \\
\hline$y$ & $\mathrm{n}$ & $\mathrm{n}$ & $\mathrm{n}$ & $y$ \\
\hline$y$ & u & $y$ & $y$ & $y$ \\
\hline$y$ & $\mathrm{n}$ & $y$ & $\mathrm{n}$ & $y$ \\
\hline y & $\mathrm{n}$ & $\mathrm{n}$ & $\mathrm{n}$ & $y$ \\
\hline $\mathrm{u}$ & $\mathrm{n}$ & $\mathrm{n}$ & $\mathrm{n}$ & $y$ \\
\hline $\mathrm{u}$ & $\mathrm{n}$ & $\mathrm{n}$ & $\mathrm{n}$ & $\mathrm{n}$ \\
\hline$y$ & $y$ & $y$ & $y$ & $y$ \\
\hline $63 \%$ & $36 \%$ & $54 \%$ & $36 \%$ & $90 \%$ \\
\hline
\end{tabular}

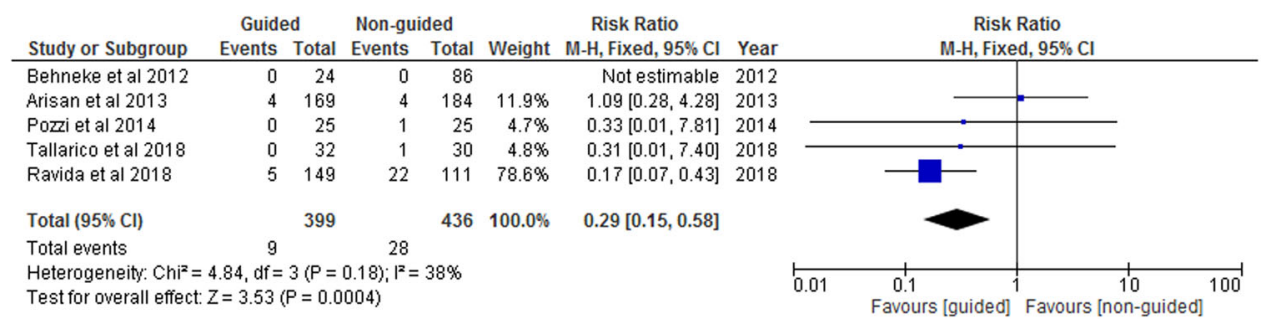

Fig. 2 Forest Plot. Forest plot comparing the "guided" group versus "non-guided" group for the event of implant failure. 


\section{REFERENCES}

1. Al-Harbi SA, Sun AY. Implant placement accuracy when using stereolithographic template as a surgical guide: preliminary results. Implant Dent. 2009;18:46-56.

2. Marlière DAA, Demétrio MS, Picinini L, De Oliveira RG, De Miranda Chaves HD. Accuracy of computer-guided surgery for dental implant placement in fully edentulous patients: a systematic review. Eur J Dent. 2018;12:153-60.

3. Wessing B, Lettner S, Zechner W. Guided bone regeneration with collagen membranes and particulate graft materials: a systematic review and metaanalysis. Int J Oral Maxillofac Implants. 2018;33:87-100.

4. Pozzi A, Tallarico M, Marchetti M, Scarfo B, Esposito M. Computer-guided versus free-hand placement of immediately loaded dental implants: 1-year post-loading results of a multicentre randomised controlled trial. Eur J Oral Implantol. 2014;7:229-42.

5. Esposito M, Maghaireh H, Grusovin MG, Ziounas I, Worthington HV. Interventions for replacing missing teeth: management of soft tissues for dental implants. Cochrane Database Syst Rev. 2012;2:Cd006697.

6. Choi W, Nguyen BC, Doan A, Girod S, Gaudilliere B, Gaudilliere D. Freehand versus guided surgery: factors influencing accuracy of dental implant placement. Implant Dent. 2017;26:500-9.

7. D' Haese J, Ackurst J, Wismeijer D, De Bruyn H, Tahmaseb A. Current state of the art of computer-guided implant surgery. Periodontology 2000. 2017;73:121-33.

8. Deshpande A, Bhargava D. Intraoral periapical radiographs with grids for implant dentistry. J Maxillofac Oral Surg. 2014;13:603-5.

9. Block MS, Robert W. Static or dynamic navigation for implant placementchoosing the method of guidance. J Oral Maxillofac Surg. 2016;74:269-77.

10. Nagarajan A, Perumalsamy $R$, Thyagarajan R, Namasivayam A. Diagnostic imaging for dental implant therapy. J Clin Imaging Sci. 2014;4:4.

11. Laverty DP, Buglass J, Patel A. Flapless dental implant surgery and use of cone beam computed tomography guided surgery. Br Dent J. 2018;224:591-602.

12. Chang El, Hanasono M. Mandibular and Maxillary Alveolar Bone Reconstruction with Free Bone Flaps and Osseointegrated Implants. In book: Vertical Alveolar Ridge Augmentation in Implant dentistry: A Surgical Manual. Wiley Online Library. 2016;275-81.

13. Karatas $\mathrm{OH}$, Toy E. Three-dimensional imaging techniques: a literature review. Eur J Dent. 2014;8:132-40.

14. Jacobs R, Salmon B, Codari M, Hassan B, Bornstein MM. Cone beam computed tomography in implant dentistry: recommendations for clinical use. BMC Oral Health. 2018;18:88.

15. Scarfe WC, Farman AG, Sukovic P. Clinical applications of cone-beam computed tomography in dental practice. JCDA. 2006;72:75-80.

16. Emery RW, Merritt SA, Lank K, Gibbs JD. Accuracy of dynamic navigation for dental implant placement-model-based evaluation. J Oral Implantol. 2016;42:399-405.

17. Gulati M, Anand V, Salaria SK, Jain N, Gupta S. Computerized implant-dentistry: advances toward automation. J Indian Soc Periodontol. 2015;19:5-10.

18. Tahmaseb A, Wismeijer D, Coucke W, Derksen W. Computer technology applications in surgical implant dentistry: a systematic review. Int J Oral Maxillofac Implants. 2014;29:25-42.

19. Arisan V, Karabuda ZC, Pis, kin B, Özdemir T. Conventional multi-slice computed tomography $(\mathrm{CT})$ and cone-beam $\mathrm{CT}(\mathrm{CBCT})$ for computer-aided implant placement. Part II: reliability of mucosa-supported stereolithographic guides. Clin Implant Dent Relat Res. 2013;15:907-17.

20. Soares MM, Harari ND, Cardoso ES, Manso MC, Conz MB, Vidigal GM,Jr. An in vitro model to evaluate the accuracy of guided surgery systems. Int J Oral Maxillofac Implants. 2012;27:824-31.

21. Bover-Ramos F, Vina-Almunia J, Cervera-Ballester J, Penarrocha-Diago M, GarciaMira B. Accuracy of implant placement with computer-guided surgery: a systematic review and meta-analysis comparing cadaver, clinical, and in vitro studies. Int J Oral Maxillo Implants. 2018;33:101-15.

22. Pjetursson $B$, Thoma $D$, Jung $R$, Zwahlen $M$, Zembic A. A systematic review of the survival and complication rates of implant-supported fixed dental prostheses (FDPs) after a mean observation period of at least 5 years. Clin Oral Implants Res. 2012;23:22-38.

23. Smith DE, Zarb GA. Criteria for success of osseointegrated endosseous implants. J Prosthet Dent. 1989;62:567-72.

24. Howe MS, Keys W, Richards D. Long-term (10-year) dental implant survival: a systematic review and sensitivity meta-analysis. J Dent. 2019;84:9-21.

25. Andreiotelli M, Kamposiora P, Papavasiliou G. Digital data management for CAD/ CAM technology. An update of current systems. Eur J Prosthet Rest Dent. 2013;21:9-15.

26. Olate S, Lyrio MCN, De Moraes M, Mazzonetto R, Moreira RWF. Influence of diameter and length of implant on early dental implant failure. J Oral Maxillofac Surg. 2010;68:414-9.

27. Buser D, Sennerby L, De Bruyn H. Modern implant dentistry based on osseointegration: 50 years of progress, current trends and open questions. Periodontology 2000. 2017;73:7-21.
28. Esposito M, Hirsch JM, Lekholm U, Thomsen P. Biological factors contributing to failures of osseointegrated oral implants. Success criteria and epidemiology. Eur J Oral Sci. 1998;106:527-51.

29. Oh Se-Lim, Shiau HJ, Rldeynos MA. Survival of dental implants at sites after implant failure: a systematic review. J Prosthet Dent. 2020;123:54-60.

30. Chrcanovic BR, Albrektsson T, Wennerberg A. Reasons for failures of oral implants. J Oral Rehab. 2014;41:443-76.

31. Tabanella G, Nowzari $H$, Slots J. Clinical and microbiological determinants of ailing dental implants. Clin Implant Dent Relat Res. 2009;11:24-36.

32. Fretwurst T, Nelson K, Tarnow DP, Wang H-L, Giannobile WV. Is metal particle release associated with peri-implant bone destruction? An emerging concept. J Dent Res. 2018;97:259-65.

33. Chappuis V, Avila-Ortiz G, Mauricio GA, Monje A. Medication-related dental implant failure: systematic review and meta-analysis. Clin Oral Impl Res. 2018;29:55-68.

34. Chrcanovic BR, Albrektsson T, Wennerberg A. Bone quality and quantity and dental implant failure: a systematic review and meta-analysis. Int J Prosthodont. 2017;30:219-37.

35. Lin G, Ye S, Liu F, He F. A retrospective study of 30,959 implants: risk factors associated with early and late implant loss. J Clin Periodontol. 2018;45:3-743.

36. Moy PK, Medina D, Shetty V, Aghaloo TL. Dental implant failure rates and associated risk factors. Int J Oral Maxillofac Implants. 2005;20:569-77.

37. McDermott NE, Chuang SK, Woo WV, Dodson TB. Complications of dental implants: identification, frequency, and associated risk factors. Int J Oral Maxillofac Implants. 2003;18:848-55.

38. Neves J, Nobre MA, Oliveira EP, Santos JM, Malo P. Risk factors for implant failure and peri-implant pathology in systemic compromised patients. J Prostho. 2018;27:409-15.

39. Propadalo I, Tranfic M, Vuka I, Barco O, Pericica TP, Puljak L. In Cochrane reviews, risk of bias assessments for allocation concealment were frequently not in line with Cochrane's Handbook guidance. J Clin Epidemiol. 2019;106:10-7.

40. Zeng X, Zhang Y, Kwong JSW, Zhang C, Li S, Sun F, et al. The methodological quality assessment tools for preclinical and clinical studies, systematic review and meta-analysis, and clinical practice guideline: a systematic review. JEBM. 2015;8:2-10.

41. Munn Z, Barker TH, Moola S, Tufanaru C, Stern C, McArthur A, et al. Methodological quality of case series studies: an introduction to the JBI critical appraisal tool. JBI Evid Synth. 2020;18:2127-33.

42. Nickenig H-J, Wichmann M, Schlegel KA, Eitner S. Evaluation of the difference in accuracy between implant placement by virtual planning data and surgical guide templates versus the conventional free-hand method-a combined in vivo-in vitro technique using cone-beam CT (Part II). Int J Oral Maxillofac Implants. 2010;7:488-93.

43. Tallarico M, Esposito M, Xhanari E, Caneva M, Meloni SM. Computer-guided vs freehand placement of immediately loaded dental implants: 5-year postloading results of a randomised controlled trial. Eur J Oral Implantol. 2018;11:203-13.

44. Arisan V, Karabuda CZ, Mumcu E, Ozdemir T. Implant positioning errors in freehands and computer-aided placement methods: a single-blinded clinical comparative study. Int J Oral Maxillofac Implants. 2013;28:190-204.

45. Behneke A, Burwinkel M, Behneke N. Factors influencing transfer accuracy of cone beam CT-derived template-based implant placement. Clin Oral Impl Res. 2012;23:416-23.

46. Danza M, Zollino I, Carinci F. Comparison between implants inserted with and without computer planning and custom model coordination. J Craniofac Surg. 2009;4:1086-92.

47. Ravidà A, Barootchi S, Tattan M, Saleh MHA, Gargallo-Albiol J, Wang H-L. Clinical outcomes and cost effectiveness of computer-guided versus conventional implant-retained hybrid prostheses: along term retrospective analysis of treatment protocols. J Periodontol. 2018;89:1015-24.

48. Yogui FC, Verri FR, de Luna Gomes JM, Lemos CAA, Cruz RS, Pellizzer EP. Comparison between computer-guided and freehand dental implant placement surgery: a systematic review and meta-analysis. Int J Oral Maxillofac Surg. 2021;50:242-50.

49. Schneider D, Marquardt $P$, Zwahlen $M$, Jung RE. A systematic review on the accuracy and the clinical outcome of computer-guided template-based implant dentistry. Clin Oral Implants Res. 2009;4:73-86.

\section{AUTHOR CONTRIBUTIONS}

MPG and SP conceived of the presented idea. MPG developed the theory and performed the computations. SP verified the analytical methods. NA investigated the research question, carried out the data analysis, wrote the manuscript with support and supervision of MPG and SP. All authors provided critical feedback and helped shape the research, analysis, and manuscript. 
COMPETING INTERESTS

The authors declare no competing interests.

\section{ADDITIONAL INFORMATION}

Correspondence and requests for materials should be addressed to M.P.G.

Reprints and permission information is available at http://www.nature.com/ reprints

Publisher's note Springer Nature remains neutral with regard to jurisdictional claims in published maps and institutional affiliations.
Open Access This article is licensed under a Creative Commons Attribution 4.0 International License, which permits use, sharing, adaptation, distribution and reproduction in any medium or format, as long as you give appropriate credit to the original author(s) and the source, provide a link to the Creative Commons license, and indicate if changes were made. The images or other third party material in this article are included in the article's Creative Commons license, unless indicated otherwise in a credit line to the material. If material is not included in the article's Creative Commons license and your intended use is not permitted by statutory regulation or exceeds the permitted use, you will need to obtain permission directly from the copyright holder. To view a copy of this license, visit http://creativecommons. org/licenses/by/4.0/.

(c) The Author(s) 2021 